\title{
Economicisation de la médecine? L'introduction des DRG dans les hôpitaux suisses - un défi éthique
}

Avec l'introduction des DRG (forfaits par cas liés au diagnostic), la Suisse se prépare à un changement du système de financement des prestations hospitalières qui soulève non seulement des questions politiques et économiques, mais également éthiques - la Commission nationale d'éthique pour la médecine humaine (NEKCNE) et l'Académie Suisse des Sciences Médicales (ASSM) se sont prononcées à ce sujet à plusieures reprises $[1,2]$. Le changement majeur réside dans le fait qu'à partir de 2012, les prestations hospitalières seront rémunérées sur la base d'un prix fixé en fonction du diagnostic et non en fonction du coût effectif des prestations. Actuellement, l'on observe un véritable boom des événements consacrés à la discussion des DRG. Pour la plupart, cependant, ceux-ci se focalisent sur les avantages des DRG d'un point de vue d'économie de la santé ou sur le débat autour des défis auxquels la mise en œuvre des systèmes DRG est confrontée. Or l'introduction des DRG est bien plus qu'une simple mesure administrative. Elle prête à s'interroger sur le rôle que joue l'hôpital au sein de notre société, sur les risques d'un appauvrissement de la qualité des soins, sur des questions d'équité quant à l'accès aux prestations ainsi que sur la protection des données médicales. Dans ce contexte, la NEK-CNE et l'ASSM ont décidé d'organiser, le 10 juin 2009, un symposium public intitulé: «Economicisation de la médecine? L'introduction des DRG dans les hôpitaux suisses - un défi éthique». Ce symposium s'intéressera particulièrement aux groupes dont le quotidien ainsi que les expériences faites à l'hôpital pourraient être influencés par l'introduction des DRG, à savoir les patients vulnérables (p. ex. les enfants) d'une part, et le personnel médical concerné (médecins et soignants) d'autre part. De même, le symposium cherchera à développer dans quelle mesure une recherche concomitante permettrait de détecter de telles influences afin de pouvoir recommander des mesures atténuantes le cas échéant. Par consé- quent, le symposium sera consacré aux questions suivantes:

- Quels sont les groupes de patients privilégiés par les systèmes des DRG? Quels sont les groupes particulièrement vulnérables? Comment protéger ces derniers de conséquences négatives?

- Quels seront les effets d'un financement basé sur les DRG sur les différents groupes professionnels et leur impact dans la médecine au quotidien?

- Quelles sont les mesures de précaution à prendre pour que le principe d'assistance et d'équité demeure garanti dans la pratique médicale et infirmière après l'introduction des DRG?

- Quelles questions doivent être examinées de façon scientifique parallèlement à l'introduction des DRG afin de prévenir toute évolution indésirable?

Les intervenants, experts issus de la pratique, du monde académique et des organisations responsables de ce symposium, seront particulièrement attentifs aux expériences récoltées jusqu'ici dans le domaine des DRG en divers endroits du pays (p. ex. au Tessin) et dans les pays limitrophes (en particulier en Allemagne). Ce colloque s'adresse aux milieux spécialisés concernés du domaine de la santé ainsi qu'au grand public. Dans la soirée, une table ronde sera organisée avec des représentants issus du monde de la politique et de la santé.

1 Prise de position no. 15/2008 de la NEK-CNE. Introduction de forfaits par cas liés au diagnostic dans les hôpitaux suisses. Bull Méd Suisses. 2008;89(36):1533-6. www.nek-cne.ch.

2 Bulletin ASSM no. 1/2009 consacré prioritairement à l'introduction des DRG en Suisse. www.samw.ch $\rightarrow$ Publications $\rightarrow$ Bulletins ASSM $\rightarrow$ $1 / 2009$. 


\section{Programme}

Symposium

Mercredi, 10 juin 2009, Auditoire Ettore Rossi, Inselspital (Hôpital des Enfants), Berne

Allocution de bienvenue et introduction au symposium. Prof. Christian Kind, St-Gall, président de la CEC de l'ASSM, et N.N., président(e) de la NEK-CNE

10.15-12.00 Patients vulnérables

Dr Bernard Braun, Brême: Der Einfluss der Einführung der DRGs auf die Situation der Patientinnen und Patienten in Deutschland

Gianni Luchessa, capo servizio amministrazione e finanze, Ente ospedaliero ticinese Locarno: Les DRGs influencent-ils la situation des patients dans les institutions? L'expérience du Tessin

Dr Michele Losa, Kinderspital St-Gall: DRGs und die spezifischen Bedürfnisse der Kinder in pädiatrischen Einrichtungen

Dr Bertrand Kiefer, membre de la NEK-CNE, Genève: L'introduction des DRG et la situation des patients dans une perspective éthique

13.15-15.15 Groupes professionnels concernés

PD Dr Arne Manzeschke, Université Bayreuth; Dr Pierre-François Cuénoud, Comité central de la FMH, Conseil d'administration SwissDRGAG, chirurgien-chef à l'hôpital cantonal de Sion: Zum Einfluss der DRGs auf Rolle und Professionsverständnis der Ärztinnen und Ärzte

Prof. Sabine Bartholomeyczik, Université Witten/Herdecke: Zum Einfluss der DRGs auf die Aufgaben und die Rolle der Pflegenden

Barbara Gassmann, vice-présidente SBK, Berne: DRGs und die Kontinuität der Pflege: Zur Arbeitsteilung zwischen stationärem und ambulantem Bereich Dr Samia Hurst, membre de la CCE de l'ASSM, Genève: L'introduction des DRG et leur influence sur le personnel médical dans une perspective éthique

$15.45 \quad$ Recherche concomitante PD Dr Simon Hölzer, directeur SwissDRG SA, Berne: Begleitforschung als Erfolgsfaktor der Einführung der DRGs Prof. Nikola Biller-Andorno et Dr Verina Wild, Universität Zürich: Anforderungen an die Begleitforschung zur Einführung der DRGs aus ethischer Sicht

17.00 Perspectives. Prof. Peter Suter, président ASSM, Genève: Rollen der Berufsgruppen, Aufgaben des Spitals: ein Blick in die Zukunft

17.30 Résumé et clôture du symposium. Dr Markus Zimmermann-Acklin, Université Lucerne, vice-président de la CEC de l'ASSM

$17.45 \quad$ Fin du symposium

19.15-20.45 Débat: opportunités et risques de l'introduction des DRG dans les hôpitaux suisses

Table ronde avec:

- Conseiller exécutif Dr Philippe Perrenoud, directeur de la santé publique du Canton de Berne (sollicité)

- Conseiller national Dr Ignazio Cassis

- Conseillère nationale Bea Heim

- Dr Jacques de Haller, président de la FMH

- Dr Bernhard Wegmüller, directeur H+ Les hôpitaux de la Suisse, Conseil d'aministration SwissDRG SA

- PD Dr Silvia Käppeli, Université de Zurich, membre de la NEK-CNE

Modération: Ellinor von Kauffungen

Langues du symposium: français et allemand (avec traduction simultanée)

Frais d'inscription: Fr. 50.-

Inscription: Académie Suisse des Sciences Médicales, Secrétariat général, Petersplatz 13, 4051 Bâle, fax 06126990 39, e-mail: mail@samw.ch 